\title{
KÄHLER IMMERSIONS OF HOMOGENEOUS KÄHLER MANIFOLDS INTO COMPLEX SPACE FORMS*
}

\author{
ANTONIO JOSE DI SCALA ${ }^{\dagger}$, HIDEYUKI ISHI ${ }^{\ddagger}$, AND ANDREA LOI ${ }^{\S}$
}

\begin{abstract}
In this paper we study the homogeneous Kähler manifolds (h.K.m.) which can be Kähler immersed into finite or infinite dimensional complex space forms. On the one hand we completely classify the h.K.m. which can be Kähler immersed into a finite or infinite dimensional complex Euclidean or hyperbolic space. On the other hand, we extend known results about Kähler immersions into the finite dimensional complex projective space to the infinite dimensional setting.
\end{abstract}

Key words. Kähler metrics, infinite dimensional complex space forms, homogeneous space, Wallach set.

AMS subject classifications. 53D05, 53C55, 58F06.

1. Introduction and statements of the main results. In this paper we address the following problem: classify all homogeneous Kähler manifolds (h.K.m. for short) which admit Kähler immersions into a given finite or infinite dimensional complex space form.

A Kähler immersion $f:(M, g) \rightarrow\left(S, g_{S}\right)$ from a Kähler manifold $(M, g)$ into a complex space form $\left(S, g_{S}\right)$ is a holomorphic map such that $f^{*} g_{S}=g$ (here $g$ and $g_{S}$ denote the Kähler metrics on $M$ and $S$ respectively).

Recall that there are three types, up to homotheties, of complex space forms $\left(S, g_{S}\right)$ according to the sign of their constant holomorphic sectional curvature:

- the complex Euclidean space $\mathbb{C}^{N}, N \leq \infty$, with the flat metric denoted by $g_{0}$. Here $\mathbb{C}^{\infty}$ is the complex Hilbert space $\ell^{2}(\mathbb{C})$ consisting of sequences $z_{j}, j=1, \ldots, z_{j} \in \mathbb{C}$ such that $\sum_{j=1}^{+\infty}\left|z_{j}\right|^{2}<+\infty$.

- the complex hyperbolic space $\mathbb{C} H^{N}, N \leq \infty$, namely the unit ball in $\mathbb{C}^{N}$ $\left(\sum_{j=1}^{N}\left|z_{j}\right|^{2}<1\right)$ endowed with the hyperbolic metric $g_{h y p}$ of holomorphic sectional curvature being -4 , whose associated Kähler form $\omega_{\text {hyp }}$ is given by:

$$
\omega_{\text {hyp }}=-\frac{i}{2} \partial \bar{\partial} \log \left(1-\sum_{j=1}^{N}\left|z_{j}\right|^{2}\right) .
$$

- the complex projective space $\mathbb{C} P^{N}, N \leq \infty$, with the Fubini-Study metric $g_{F S}$ of holomorphic sectional curvature being 4 . If $\omega_{F S}$ denotes the Kähler form associated to $g_{F S}$ and $\left[Z_{0}, \ldots, Z_{N}\right]$ are homogeneous coordinates on $\mathbb{C} P^{N}$, then in the affine chart $U_{k}=\left\{Z_{k} \neq 0\right\}, k=0, \ldots, N$, $\omega_{F S}=\frac{i}{2} \partial \bar{\partial} \log \left(1+\sum_{j \neq k}\left|Z_{j} / Z_{k}\right|^{2}\right)$. If $\pi: \mathbb{C}^{N+1} \backslash\{0\} \ni\left(Z_{0}, \ldots, Z_{N}\right) \mapsto$

${ }^{*}$ Received August 17, 2011; accepted for publication October 13, 2011. Research partially supported by GNSAGA (INdAM), KAKENHI19740070 (JSPS) and MIUR (PRIN07, Differential Geometry and Global Analysis).

$\dagger$ Dipartimento di Scienze Matematiche, Politecnico di Torino, Corso Duca degli Abruzzi 24, 10129 Torino, Italy (antonio.discala@polito.it). The first author was (partially) supported by ESF within the program "Contact and Symplectic Topology".

$\ddagger$ Graduate School of Mathematics, Nagoya University, Furo-cho, 464-8602 Nagoya, Japan (hideyuki@math.nagoya-u.ac.jp).

§Dipartimento di Matematica e Informatica, Università di Cagliari, Via Ospedale 72, 09124 Cagliari, Italy (loi@unica.it). 
$\left[Z_{0}, \ldots, Z_{N}\right] \in \mathbb{C} P^{N}$ is the canonical projection, $\omega_{F S}$ is also characterized by the relation $\pi^{*} \omega_{F S}=\frac{i}{2} \partial \bar{\partial} \log \left(\sum_{j=0}^{N}\left|Z_{j}\right|^{2}\right)$.

Notation. When we speak about the Kähler manifold $\mathbb{C}^{N}\left(\right.$ resp. $\mathbb{C} H^{N}$ or $\left.\mathbb{C} P^{N}\right)$ without mentioning the Kähler metric we will always mean $\mathbb{C}^{N}$ (resp. $\mathbb{C} H^{N}$ or $\mathbb{C} P^{N}$ ) equipped with the metric $g_{0}$ (resp. $g_{h y p}, g_{F S}$ ).

Note that, once that a Kähler immersion into a complex space form $\left(S, g_{S}\right)$ is given, then all other Kähler immersions can be obtained by composing it with a unitary transformation of $\left(S, g_{S}\right)$. This is due to the following celebrated rigidity theorem due to E. Calabi [Ca53] which will be of constant use throughout this paper.

Theorem (CAlabi's Rigidity theorem). Let $f:(M, g) \rightarrow\left(S, g_{S}\right)$ and $\tilde{f}$ : $(M, g) \rightarrow\left(S, g_{S}\right)$ be two Kähler immersions into the same complex space form $\left(S, g_{S}\right)$. Then there exists a unitary transformation $U$ of $\left(S, g_{S}\right)$ such that $f=U \circ \tilde{f}$.

1.1. Immersions in $\mathbb{C}^{N}$ and $\mathbb{C} H^{N}$. In the following two theorems we give a complete solution of our problem when the ambient space is $\mathbb{C}^{N}$ or $\mathbb{C} H^{N}, N \leq \infty$. In order to state our result note that the map $f_{n}: \mathbb{C} H^{n} \rightarrow l^{2}(\mathbb{C})$ given by:

$$
z=\left(z_{1}, \ldots z_{n}\right) \stackrel{f_{n}}{\mapsto}\left(\ldots, \sqrt{\frac{(|j|-1) !}{j !}} z_{1}^{j_{1}} \ldots z_{n}^{j_{n}}, \ldots\right)
$$

is a Kähler immersion of $\mathbb{C} H^{n}$ into $l^{2}(\mathbb{C})$, i.e. $f_{n}^{*} g_{0}=g_{h y p}$, (see [Ca53]), where $|j|=j_{1}+\cdots+j_{n}$ and $j !=j_{1} ! \cdots j_{n} !$

TheOREM 1. Let $(M, g)$ be a n-dimensional h.K.m..

(a) If $(M, g)$ can be Kähler immersed into $\mathbb{C}^{N}, N<\infty$, then $(M, g)=\mathbb{C}^{n}$;

(b) if $(M, g)$ can be Kähler immersed into $\ell^{2}(\mathbb{C})$, then $(M, g)$ equals

$$
\mathbb{C}^{k} \times \mathbb{C} H_{\lambda_{1}}^{n_{1}} \times \cdots \times \mathbb{C} H_{\lambda_{r}}^{n_{r}}
$$

where $k+n_{1}+\cdots+n_{r}=n, \lambda_{j}, j=1, \ldots, r$ are positive real numbers and $\mathbb{C} H_{\lambda_{j}}^{n_{j}}=\left(\mathbb{C} H^{n_{j}}, \lambda_{j} g_{\text {hyp }}\right), j=1, \ldots, r$ (hence $\left.\mathbb{C} H_{1}^{n}=\mathbb{C} H^{n}\right)$.

Moreover, in case (a) (resp. case (b)) the immersion is given, up to a unitary transformation of $\mathbb{C}^{N}$ (resp. $\ell^{2}(\mathbb{C})$ ), by the linear inclusion $\mathbb{C}^{n} \hookrightarrow \mathbb{C}^{N}$ (resp. by $\left(f_{0}, f_{1}, \ldots, f_{r}\right)$, where $f_{0}$ the linear inclusion $\mathbb{C}^{k} \hookrightarrow \ell^{2}(\mathbb{C})$ and each $f_{j}: \mathbb{C} H^{n_{j}} \rightarrow \ell^{2}(\mathbb{C})$ is $\sqrt{\lambda_{j}}$ times the map (2)).

Theorem 2. Let $(M, g)$ be a n-dimensional h.K.m.. Then if $(M, g)$ can be Kähler immersed into $\mathbb{C} H^{N}, N \leq \infty$, then $(M, g)=\mathbb{C} H^{n}$ and the immersion is given, up to a unitary transformation of $\mathbb{C} H^{N}$, by the linear inclusion $\mathbb{C} H^{n} \hookrightarrow \mathbb{C} H^{N}$

REMARK 1. Since a Kähler immersion is minimal, an alternative proof of (1) in Theorem 1 when $N<\infty$ follows by the work of A. J. Di Scala [DS02].

Remark 2. Assertion (2) in Theorem 1 is a generalization to arbitrary h.K.m. of Theorem 3.3 in [DL07] where the first and the third authors proved that a bounded symmetric domain which can be Kähler immersed into $\ell^{2}(\mathbb{C})$ is necessarily of rank one. Actually, the method of the present paper, when applied to bounded symmetric domains, provides us with an alternative and more elegant proof of this result (cfr. Remark 7 below). 
1.2. Immersion in $\mathbb{C} P^{N}$. There exists a large class (cfr. Conjecture 1 below) of h.K.m. which can be Kähler immersed into $\mathbb{C} P^{N}$. In this paper a Kähler metric $g$ on a complex manifold $M$ will be called projectively induced if there exists an immersion $f: M \rightarrow \mathbb{C} P^{N}, N \leq \infty$, such that $f^{*} g_{F S}=g$. An obvious necessary condition for $g$ to be projectively induced is that its associated Kähler form $\omega$ is integral i.e. it represents the first Chern class $c_{1}(L)$ in $H^{2}(M, \mathbb{Z})$ of a holomorphic line bundle $L \rightarrow M$. Indeed $L$ can be taken as the pull-back of the hyperplane line bundle on $\mathbb{C} P^{N}$ whose first Chern class is given by $\omega_{F S}$. Notice that if $\omega$ is an exact form (e.g. when $M$ is contractible) then $\omega$ is obviously integral since its second cohomology class vanishes.

Other (less obvious) conditions are expressed by the following theorem and its corollary which represent our first result about projectively induced Kähler metrics.

TheOREM 3. Assume that a h.K.m. $(M, g)$ admits a Kähler immersion $f: M \rightarrow$ $\mathbb{C} P^{N}, N \leq \infty$. Then $M$ is simply-connected and $f$ is injective.

Corollary 3. Let $(M, g)$ be a complete and locally h.K.m.. Assume that $f$ : $(M, g) \rightarrow \mathbb{C} P^{N}, N \leq \infty$, is a Kähler immersion. Then $(M, g)$ is a h.K.m..

When the dimension of the ambient space is finite, i.e. $\left(S, g_{S}\right)=\mathbb{C} P^{N}, N<\infty$, $M$ is forced to be compact and a proof of Theorem 3 is well-known by the work of M. Takeuchi [TA78]. In this case he also provides a complete classification of all compact h.K.m. which can be Kähler immersed into $\mathbb{C} P^{N}$ by making use of the representation theory of semisimple Lie groups. Viceversa, it is not hard to see that if a compact Kähler manifold can be Kähler immersed into $\mathbb{C} P^{\infty}$ then it can also be Kähler immersed into $\mathbb{C} P^{N}$ with $N<\infty$.

We believe that, up to homotheties, any simply-connected h.K.m. such that its associated Kähler form is integral can be Kähler immersed into $\mathbb{C} P^{N}$, with $N \leq \infty$. This is expressed by the following conjecture.

CONJECTURE 1. Let $(M, g)$ be a simply-connected h.K.m. such that its associated Kähler form $\omega$ is integral. Then there exists $\lambda_{0} \in \mathbb{R}^{+}$such that $\lambda_{0} g$ is projectively induced.

The integrality of $\omega$ in the conjecture is important since there exist simplyconnected h.K.m. $(M, \omega)$ such that $\lambda \omega$ is not integral for any $\lambda \in \mathbb{R}^{+}$(take, for example, $\left.(M, g)=\left(\mathbb{C} P^{1}, g_{F S}\right) \times\left(\mathbb{C} P^{1}, \sqrt{2} g_{F S}\right)\right)$. Observe also that there exist simplyconnected (even contractible) h.K.m. $(M, g)$ such that $\omega$ is an integral form but $g$ is not projectively induced. In order to describe such an example we recall the following result (see Theorem 2 in [LZ11]).

TheOREM A.Let $g_{B}$ be the Bergman metric of an irreducible Hermitian symmetric space of noncompact type $\Omega$. Then $\lambda g_{B}$ is projectively induced if and only $\lambda \gamma$ belongs to $W(\Omega) \backslash\{0\}$, where $\gamma$ denotes the genus of $\Omega$ and $W(\Omega)$ its Wallach set.

It turns out (see Corollary 4.4 p. 27 in [AR95] and references therein) that $W(\Omega)$ consists only of real numbers and depends on two of the domain's invariants, denoted by $a$ (strictly positive natural number) and $r$ (the rank of $\Omega$ ). More precisely we have

$$
W(\Omega)=\left\{0, \frac{a}{2}, 2 \frac{a}{2}, \ldots,(r-1) \frac{a}{2}\right\} \cup\left((r-1) \frac{a}{2}, \infty\right) .
$$


The set $W_{d}=\left\{0, \frac{a}{2}, 2 \frac{a}{2}, \ldots,(r-1) \frac{a}{2}\right\}$ and the interval $W_{c}=\left((r-1) \frac{a}{2}, \infty\right)$ are called respectively the discrete and continuous part of the Wallach set of the domain $\Omega$. Observe that when $r=1$, namely $\Omega$ is the complex hyperbolic space $\mathbb{C} H^{n}$, then $g_{B}=(n+1) g_{\text {hyp }}$. In this case (and only in this case) $W_{d}=\{0\}$ and $W_{c}=(0, \infty)$. If $\operatorname{rank}(\Omega)=r \geq 2$ and $0<\lambda<\frac{a}{2 \gamma}$ it follows by Theorem A that $\lambda g_{B}$ is not projectively induced and its associated Kähler form $\lambda \omega_{B}$ is integral (since $\Omega$ is contractible). This provides us with the desired example.

Notice also that from Theorem A it follows that the only irreducible bounded symmetric domain where $\lambda g_{B}$ is projectively induced for all $\lambda>0$ is the complex hyperbolic space. In the following theorem, which represents our last result, we generalize this fact to any homogeneous bounded domain (h.b.d. for short). This will be a key ingredient in the proof of Theorem 1.

THEOREM 4. Let $(\Omega, g)$ be a n-dimensional h.b.d.. The metric $\lambda g$ is projectively induced for all $\lambda>0$ if and only if

$$
(\Omega, g)=\mathbb{C} H_{\lambda_{1}}^{n_{1}} \times \cdots \times \mathbb{C} H_{\lambda_{r}}^{n_{r}},
$$

where $n_{1}+\cdots+n_{r}=n, \lambda_{j}, j=1, \ldots, r$ are positive real numbers and $\mathbb{C} H_{\lambda_{j}}^{n_{j}}=$ $\left(\mathbb{C} H^{n_{j}}, \lambda_{j} g_{\text {hyp }}\right), j=1, \ldots, r$.

The paper contains another section dedicated to the proofs of our main results.

Aknowledgments. The second and third author would like to thank Politecnico of Torino for the wonderful hospitality in their research stays in January 2010.

2. Proof of the main results. The basic ingredient for the proof of our results is the following solution due to J. Dorfmeister and K. Nakajima [DN88] of the fundamental conjecture on h.K.m.

ThEOREM FC. A h.K.m. $(M, g)$ is the total space of a holomorphic fiber bundle over a h.b.d. $\Omega$ in which the fiber $\mathcal{F}=\mathcal{E} \times \mathcal{C}$ is (with the induced Kähler metric) the Kähler product of a flat homogeneous Kähler manifold $\mathcal{E}$ and a compact simplyconnected homogeneous Kähler manifold $\mathcal{C}$.

In order to prove Theorem 1 recall that complete connected totally geodesic submanifolds of $\mathbb{R}^{n}$ are affine subspaces $p+\mathbb{W}$, where $p \in \mathbb{R}^{n}$ and $\mathbb{W} \subset \mathbb{R}^{n}$ is a vector subspace. We need the following result from [AD03].

LEMMA 4. Let $G$ be a connected Lie subgroup of isometries of the Euclidean space $\mathbb{R}^{n}$. Let $G . p=p+\mathbb{V}$ and $G . q=q+\mathbb{W}$ be two totally geodesic $G$-orbits. Then $\mathbb{V}=\mathbb{W}$, i.e. G.p and $G . q$ are parallel affine subspaces of $\mathbb{R}^{n}$.

We also need the following lemmata. The first one due to Bochner (see Theorem 14 in [Bo47]) shows that if a Kähler manifolds can be Kähler immersed into a finite or infinite complex flat space then it can be Kähler immersed into the infinite dimensional complex projective space. The second one due to the first and the third authors of the present paper (see Lemma 3.1 in [DL07]) is a splitting result for maps into complex Euclidean spaces.

Lemma 5. Assume that a Kähler manifold $(M, g)$ can be Kähler immersed into $\mathbb{C}^{N}, N \leq \infty$. Then there exists a Kähler immersion of $(M, g)$ into $\mathbb{C} P^{\infty}$. 
Lemma 6. Let $f: M \times M^{\prime} \rightarrow \mathbb{C}^{N}, N \leq \infty$, be a Kähler immersion from a product $M \times M^{\prime}$ of two Kähler manifolds. Then $f$ is a product, i.e. $f(p, q)=\left(f_{1}(p), f_{2}(q)\right)$ where $f_{1}: M \rightarrow \mathbb{C}^{N_{1}}$ and $f_{2}: M^{\prime} \rightarrow \mathbb{C}^{N_{2}}$ are Kähler immersions with $N_{1}+N_{2}=N$.

We are now in the position to prove Theorem 1 which, as we have already pointed out in the introduction, will be proved assuming the validity of Theorem 4 .

Proof of Theorem 1. Assume that there exists a Kähler immersion $f: M \rightarrow \mathbb{C}^{N}$. By Theorem FC and by the fact that a h.b.d. is contractible we get that $M=\mathbb{C}^{k} \times \Omega$ as a complex manifold since, by the maximum principle, the fiber $\mathcal{F}$ cannot contain a compact manifold. Let $M=G / K$ be the homogeneous realization of $M$ (so the metric $g$ is $G$-invariant). It follows again by Theorem FC that there exists $L \subset G$ such that the $L$-orbits are the fibers of the fibration $\pi: M=G / K \rightarrow \Omega=G / L$. Let $F_{p}, F_{q}$ be the fibers over $p, q \in \Omega$. We claim that $f\left(F_{p}\right)$ and $f\left(F_{q}\right)$ are parallel affine subspaces of $\mathbb{C}^{N}$. Indeed, by Calabi's rigidity $f\left(F_{p}\right)$ and $f\left(F_{q}\right)$ are affine subspaces of $\mathbb{C}^{N}$ since both $F_{p}$ and $F_{q}$ are flat Kähler manifolds of $\mathbb{C}^{n}$. Moreover, Calabi rigidity theorem implies the existence of a morphism of groups $\rho: G \rightarrow I s o_{\mathbb{C}}\left(\mathbb{C}^{N}\right)=\mathrm{U}\left(\mathbb{C}^{N}\right) \ltimes \mathbb{C}^{N}$ such that $f(g \cdot x)=\rho(g) f(x)$ for all $g \in G, x \in M$. Let $W_{p, q}$ be the affine subspace generated by $f\left(F_{p}\right)$ and $f\left(F_{q}\right)$. Since both $f\left(F_{p}\right)$ and $f\left(F_{q}\right)$ are $\rho(L)$-invariant it follows that $W_{p, q}$ is also $\rho(L)$-invariant. Indeed, for any $g \in L$ the isometry $\rho(g)$ is an affine map and so must preserve the affine space generated by $f\left(F_{p}\right)$ and $f\left(F_{q}\right)$. Observe that $W_{p, q}$ is a finite dimensional complex Euclidean space, $\rho(L)$ acts on $W_{p, q}$ and $f\left(F_{p}\right)$ and $f\left(F_{q}\right)$ are two complex totally geodesic orbits in $W_{p, q}$. Then, by Lemma 4 , we get that $f\left(F_{p}\right)$ and $f\left(F_{q}\right)$ are parallel affine subspaces of $W_{p, q}$ and hence of $\mathbb{C}^{N}$. Since $p, q \in \Omega$ are two arbitrary points it follows that $f(M)$ is a Kähler product. Thus $M=\mathbb{C}^{k} \times \Omega$ is a Kähler product of homogeneous Kähler manifolds. Using again the fact $M$ can be Kähler immersed into $\mathbb{C}^{N}$ it follows that the h.b.d. $\Omega$ can be Kähler immersed into $\mathbb{C}^{N}$. If one denotes by $\varphi$ this immersion and by $g_{\Omega}$ the homogeneous Kähler metric of $\Omega$, it follows that the map $\sqrt{\lambda} \varphi$ is a Kähler immersion of $\left(\Omega, \lambda g_{\Omega}\right)$ into $\mathbb{C}^{N}$. Therefore, by Lemma $5, \lambda g_{\Omega}$ is projectively induced for all $\lambda>0$ and Theorem 4 yields

$$
(M, g)=\mathbb{C}^{k} \times \mathbb{C} H_{\lambda_{1}}^{n_{1}} \times \cdots \times \mathbb{C} H_{\lambda_{r}}^{n_{r}},
$$

where $k+n_{1}+\cdots+n_{r}=n$ and $\lambda_{j}, j=1, \ldots, r$ are positive real numbers. If the dimension $N$ of the ambient space $\mathbb{C}^{N}$ is finite then $M=\mathbb{C}^{n}$ since there cannot exist a Kähler immersion of $\left(\mathbb{C} H^{n_{j}}, \lambda_{j} g_{h y p}\right)$ into $\mathbb{C}^{N}, N<\infty$ (see [Ca53]) and this proves (a). The last part of Theorem 1 is a consequence of Calabi's rigidity theorem together with Lemma 6.

REMARK 7. As we have already pointed, Theorem 4, which is an important step in the proof of the Theorem 1, is a straightforward consequence of Theorem A above when the h.K.m. is a bounded symmetric domain. Therefore the last part of Theorem 1 provides an alternative proof of Theorem 3.3 in [DL07] without the use of Calabi's diastasis function (cfr. Remark 2).

In order to prove Theorem 2 we need the following lemma.

LEMMA 8. If a Kähler manifold $(M, g)$ can be Kähler immersed into $\mathbb{C} H^{N}$, $N \leq \infty$, then it can also be Kähler immersed into $\ell^{2}(\mathbb{C})$.

Proof. Let $f$ be the Kähler immersion of $(M, g)$ into $\mathbb{C} H^{N}$. If $N<\infty$ then the map $f_{n} \circ f:(M, g) \rightarrow \ell^{2}(\mathbb{C})$, where $f_{n}$ is given by $(2)$, is a Kähler immersion. 
If $N=\infty$, it follows by (1) in the introduction that $\Phi=-\log \left(1-\sum_{j=1}^{\infty}\left|\phi_{j}\right|^{2}\right)=$ $\sum_{k=1}^{\infty}\left(\sum_{j=1}^{\infty}\left|\phi_{j}\right|^{2}\right)^{k} / k$ is a Kähler potential for the metric $g$, i.e. $\frac{i}{2} \partial \bar{\partial} \Phi=\omega$, where $\omega$ is the Kähler form associated to the metric $g$ and the $\phi_{j}$ 's are the components of $f$. Hence $\Phi=\sum_{j=1}^{\infty}\left|h_{j}\right|^{2}$ for suitable holomorphic functions $h_{j}, j=1,2, \ldots$ on $M$ and the map $h=\left(\ldots, h_{j}, \ldots\right):(M, g) \rightarrow \ell^{2}(\mathbb{C})$ is the desired Kähler immersion.

Proof of Theorem 2. If a h.K.m. $(M, g)$ can be Kähler immersed into $\mathbb{C} H^{N}$, $N \leq \infty$, then, by Lemma 8 it can also be Kähler immersed into $\ell^{2}(\mathbb{C})$. By Theorem $1,(M, g)$ is then a Kähler product of complex space forms, namely

$$
(M, g)=\mathbb{C}^{k} \times \mathbb{C} H_{\lambda_{1}}^{n_{1}} \times \cdots \times \mathbb{C} H_{\lambda_{r}}^{n_{r}} .
$$

Then the conclusion follows from the fact that $\mathbb{C}^{k}$ cannot be Kähler immersed into $\mathbb{C} H^{N}$ for all $N \leq \infty$ (see [Ca53]), from Calabi's rigidity theorem and from Theorem 2.11 in [AD03] which shows that there are not Kähler immersions from a product $M \times M^{\prime}$ of Kähler manifolds into $\mathbb{C} H^{N}, N \leq \infty$, (the proof in [AD03] is given for $N<\infty$ but it extends without any substantial change to the infinite dimensional case).

Proof of Theorem 3. Theorem FC and the fact that a h.b.d. is contractible imply that $M$ is a complex product $\Omega \times \mathcal{F}$, where $\mathcal{F}=\mathcal{E} \times \mathcal{C}$ is a Kähler product of a flat Kähler manifold $\mathcal{E}$ Kähler embedded into $(M, g)$ and a simply-connected h.K.m. $\mathcal{C}$. We claim that $\mathcal{E}$ is simply-connected and hence $M=\Omega \times \mathcal{E} \times \mathcal{C}$ is simply-connected. In order to prove our claim notice that $\mathcal{E}$ is the Kähler product $\mathbb{C}^{k} \times T_{1} \times \cdots \times T_{s}$, where $T_{j}$ are flat complex tori. So one needs to show that each $T_{j}$ reduces to a point. If, by a contradiction, the dimension of one of this tori, say $T_{j_{0}}$ is not zero, then by composing the Kähler immersion of $T_{j_{0}}$ in $(M, g)$ with the immersion $f: M \rightarrow \mathbb{C} P^{N}$ we would get a Kähler immersion of $T_{j_{0}}$ into $\mathbb{C} P^{N}$ in contrast with a well-known result of Calabi [Ca53] (see also Lemma 2.2 in [TA78]). In order to prove that $f$ is injective we first observe that, by Calabi's rigidity theorem, $f(M)$ is still a h.K.m.. Then, by the first part of the theorem, $f(M) \subset \mathbb{C} P^{N}$ is simply-connected. Moreover, since $M$ is complete and $f: M \rightarrow f(M)$ is a local isometry, it is a covering map (see, e.g., Lemma 3.3 p. 150 in [DC92]) and hence injective.

Proof of Corollary 3. Let $\pi: \tilde{M} \rightarrow M$ be the universal covering map. Then $(\tilde{M}, \tilde{g})$ is a h.K.m. and, by Theorem $3, f \circ \pi: \tilde{M} \rightarrow \mathbb{C} P^{n}$ is injective. Therefore $\pi$ is injective, and since it is a covering map, it defines a holomorphic isometry between $(\tilde{M}, \tilde{g})$ and $(M, g)$.

Proof of Theorem 4. First we find a global potential of the homogeneous Kähler metric $g$ on the domain $\Omega$ following Dorfmeister [D85]. By [D85, Theorem 2 (c)], there exists a split solvable Lie subgroup $S \subset \operatorname{Aut}(\Omega, g)$ acting simply transitively on the domain $\Omega$. Taking a reference point $z_{0} \in \Omega$, we have a diffeomorphism $S \ni s \stackrel{\sim}{\mapsto}$ $s \cdot z_{0} \in \Omega$, and by the differentiation, we get the linear isomorphism $\mathfrak{s}:=\operatorname{Lie}(S) \ni$ $X \stackrel{\sim}{\mapsto} X \cdot z_{0} \in T_{z_{0}} \Omega \equiv \mathbb{C}^{n}$. Then the evaluation of the Kähler form $\omega$ on $T_{z_{0}} \Omega$ is given by $\omega\left(X \cdot z_{0}, Y \cdot z_{0}\right)=\beta([X, Y])(X, Y \in \mathfrak{s})$ with a certain linear form $\beta \in \mathfrak{s}^{*}$. Let $j: \mathfrak{s} \rightarrow \mathfrak{s}$ be the linear map defined in such a way that $(j X) \cdot z_{0}=\sqrt{-1}\left(X \cdot z_{0}\right)$ for $X \in \mathfrak{s}$. We have $\Re g\left(X \cdot z_{0}, Y \cdot z_{0}\right)=\beta([j X, Y])$ for $X, Y \in \mathfrak{s}$, and the right-hand side defines a positive inner product on $\mathfrak{s}$. Let $\mathfrak{a}$ be the orthogonal complement of $[\mathfrak{s}, \mathfrak{s}]$ in $\mathfrak{s}$ with respect to the inner product. Then $\mathfrak{a}$ is a commutative Cartan subalgebra of $\mathfrak{s}$. Define $\gamma \in \mathfrak{a}^{*}$ by $\gamma(C):=-4 \beta(j C)(C \in \mathfrak{a})$, and we extended $\gamma$ to $\mathfrak{s}=\mathfrak{a} \oplus[\mathfrak{s}, \mathfrak{s}]$ by 
the zero-extension. Keeping the diffeomorphism between $S$ and $\Omega$ in mind, we define a positive smooth function $\Psi$ on $\Omega$ by

$$
\Psi\left((\exp X) \cdot z_{0}\right)=e^{-\gamma(X)}(X \in \mathfrak{s}) .
$$

From the argument in [D85, pp. 302-304], we see that

$$
\omega=\frac{i}{2} \partial \bar{\partial} \log \Psi \text {. }
$$

It is known that there exists a unique kernel function $\tilde{\Psi}: \Omega \times \Omega \rightarrow \mathbb{C}$ such that (1) $\tilde{\Psi}(z, z)=\Psi(z)$ for $z \in \Omega$ and (2) $\tilde{\Psi}(z, w)$ is holomorphic in $z$ and anti-holomorphic in $w$ (cf. [I99, Proposition 4.6]). Let us observe that the metric $g$ is projectively induced if and only if $\tilde{\Psi}$ is a reproducing kernel of a Hilbert space of holomorphic functions on $\Omega$. Indeed, if $f: \Omega \rightarrow \mathbb{C} P^{N}(N \leq \infty)$ is a Kähler immersion with $f(z)=$ $\left[\psi_{0}(z): \psi_{1}(z): \cdots\right](z \in \Omega)$ its homogeneous coordinate expression, then we have $\omega=\frac{i}{2} \partial \bar{\partial} \log \sum_{j=0}^{N}\left|\psi_{j}\right|^{2}$. Comparing (5) with it, we see that there exists a holomorphic function $\phi$ on $\Omega$ for which $\Psi=\left|e^{\phi}\right|^{2} \sum_{j=0}^{N}\left|\psi_{j}\right|^{2}$. By analytic continuation, we obtain $\tilde{\Psi}(z, w)=e^{\phi(z)} \overline{e^{\phi(w)}} \sum_{j=0}^{N} \psi_{j}(z) \overline{\psi_{j}(w)}$ for $z, w \in \Omega$. For any $z_{1}, \ldots, z_{m} \in \Omega$ and $c_{1}, \ldots, c_{m} \in \mathbb{C}$, we have

$$
\begin{aligned}
\sum_{p, q=1}^{m} c_{p} \bar{c}_{q} \tilde{\Psi}\left(z_{p}, z_{q}\right) & =\sum_{p, q=1}^{m} c_{p} \bar{c}_{q} e^{\phi\left(z_{p}\right)} \overline{e^{\phi\left(z_{q}\right)}} \sum_{j=0}^{N} \psi_{j}\left(z_{p}\right) \overline{\psi_{j}\left(z_{q}\right)} \\
& =\sum_{j=0}^{N}\left|\sum_{p=1}^{m} c_{p} e^{\phi\left(z_{p}\right)} \psi_{j}\left(z_{p}\right)\right|^{2} \geq 0 .
\end{aligned}
$$

Thus the matrix $\left(\tilde{\Psi}\left(z_{p}, z_{q}\right)\right)_{p, q} \in \operatorname{Mat}(m, \mathbb{C})$ is always a positive Hermitian matrix. Therefore $\tilde{\Psi}$ is a reproducing kernel of a Hilbert space (see [Ar50, p. 344]).

On the other hand, if $\tilde{\Psi}$ is a reproducing kernel of a Hilbert space $\mathcal{H} \subset \mathcal{O}(\Omega)$, then by taking an orthonormal basis $\left\{\psi_{j}\right\}_{j=0}^{N}$ of $\mathcal{H}$, we have a Kähler immersion $f: M \ni$ $z \mapsto\left[\psi_{0}(z): \psi_{1}(z): \cdots\right] \in \mathbb{C} P^{N}$ because we have $\Psi(z)=\tilde{\Psi}(z, z)=\sum_{j=0}^{N}\left|\psi_{j}(z)\right|^{2}$. Note that there exists no point $a \in \Omega$ such that $\psi_{j}(a)=0$ for all $1 \leq j \leq N$ since $\Psi(z)=\sum_{j=0}^{N}\left|\psi_{j}(z)\right|^{2}$ is always positive.

The condition for $\tilde{\Psi}$ to be a reproducing kernel is described in [199]. In order to apply the results, we need a fine description of the Lie algebra $\mathfrak{s}$ with $j$ due to Piatetskii-Shapiro [PS69]. Indeed, it is shown in [PS69, Chapter 2] that the correspondence between the h.b.d. $\Omega$ and the structure of $(\mathfrak{s}, j)$ is one-to-one up to natural equivalence. For a linear form $\alpha$ on the Cartan algebra $\mathfrak{a}$, we denote by $\mathfrak{s}_{\alpha}$ the root subspace $\{X \in \mathfrak{s} ;[C, X]=\alpha(C) X(\forall C \in \mathfrak{a})\}$ of $\mathfrak{s}$. The number $r:=\operatorname{dim} \mathfrak{a}$ is nothing but the rank of $\Omega$. Thanks to [PS69, Chapter 2, Section 3], there exists a basis $\left\{\alpha_{1}, \ldots, \alpha_{r}\right\}$ of $\mathfrak{a}^{*}$ such that $\mathfrak{s}=\mathfrak{s}(0) \oplus \mathfrak{s}(1 / 2) \oplus \mathfrak{s}(1)$ with

$$
\begin{aligned}
\mathfrak{s}(0) & =\mathfrak{a} \oplus \sum_{1 \leq k<l \leq r}^{\oplus} \mathfrak{s}_{\left(\alpha_{l}-\alpha_{k}\right) / 2}, \quad \mathfrak{s}(1 / 2)=\sum_{1 \leq k \leq r}^{\oplus} \mathfrak{s}_{\alpha_{k} / 2}, \\
\mathfrak{s}(1) & =\sum_{1 \leq k \leq r}^{\oplus} \mathfrak{s}_{\alpha_{k}} \oplus \sum_{1 \leq k<l \leq r}^{\oplus} \mathfrak{s}_{\left(\alpha_{l}+\alpha_{k}\right) / 2} .
\end{aligned}
$$

If $\left\{A_{1}, \ldots, A_{r}\right\}$ is the basis of $\mathfrak{a}$ dual to $\left\{\alpha_{1}, \ldots, \alpha_{r}\right\}$, then $\mathfrak{s}_{\alpha_{k}}=\mathbb{R} j A_{k}$. Thus $\mathfrak{s}_{\alpha_{k}}(k=$ $1, \ldots, r)$ is always one dimensional, whereas other root spaces $\mathfrak{s}_{\alpha_{k} / 2}$ and $\mathfrak{s}_{\left(\alpha_{l} \pm \alpha_{k}\right) / 2}$ 
may be $\{0\}$. Since $\left\{\alpha_{1}, \ldots, \alpha_{r}\right\}$ is a basis of $\mathfrak{a}^{*}$, the linear form $\gamma \in \mathfrak{a}^{*}$ is written as $\gamma=\sum_{k=1}^{r} \gamma_{k} \alpha_{k}$ with unique $\gamma_{1}, \ldots, \gamma_{r} \in \mathbb{R}$. Since $j A_{k} \in \mathfrak{s}_{\alpha_{k}}$, we have

$$
\gamma_{k}=\gamma\left(A_{k}\right)=-4 \beta\left(j A_{k}\right)=-4 \beta\left(\left[A_{k}, j A_{k}\right]\right)=4 \beta\left(\left[j A_{k}, A_{k}\right]\right)
$$

and the last term equals $4 g\left(A_{k} \cdot z_{0}, A_{k} \cdot z_{0}\right)$. Thus we get $\gamma_{k}>0$.

For $\epsilon=\left(\epsilon_{1}, \ldots, \epsilon_{r}\right) \in\{0,1\}^{r}$, put $q_{k}(\epsilon):=\sum_{l>k} \epsilon_{l} \operatorname{dim} \mathfrak{s}_{\left(\alpha_{l}-\alpha_{k}\right) / 2}(k=1, \ldots, r)$. Define

$$
\mathfrak{X}(\epsilon):=\left\{\left(\sigma_{1}, \ldots, \sigma_{r}\right) \in \mathbb{C}^{r} ; \begin{array}{cc}
\sigma_{k}>q_{k}(\epsilon) / 2 & \left(\epsilon_{k}=1\right) \\
\sigma_{k}=q_{k}(\epsilon) / 2 & \left(\epsilon_{k}=0\right)
\end{array}\right\}
$$

and $\mathfrak{X}:=\bigsqcup_{\epsilon \in\{0,1\}^{r}} \mathfrak{X}(\epsilon)$. By [I99, Theorem 4.8], $\tilde{\Psi}$ is a reproducing kernel if and only if $\gamma:=\left(\gamma_{1}, \ldots, \gamma_{r}\right)$ belongs to $\mathfrak{X}$. We denote by $W(g)$ the set of $\lambda>0$ for which $\lambda g$ is projectively induced. Since the metric $\lambda g$ corresponds to the parameter $\lambda \underline{\gamma}$, we see that $\lambda g$ is projectively induced if and only if $\lambda \underline{\gamma} \in \mathfrak{X}$. Namely we obtain

$$
W(g)=\{\lambda>0 ; \lambda \underline{\gamma} \in \mathfrak{X}\},
$$

and the right-hand side is considered in [I11]. Put $q_{k}=\sum_{l>k} \operatorname{dim} \mathfrak{s}_{\left(\alpha_{l}-\alpha_{k}\right) / 2}$ for $k=1, \ldots, r$. Then [I11, Theorem 15] tells us that

$$
W(g) \cup\{0\} \subset\left\{\frac{q_{k}}{2 \gamma_{k}} ; k=1, \ldots, r\right\} \cup\left(c_{0},+\infty\right),
$$

where $c_{0}:=\max \left\{\frac{q_{k}}{2 \gamma_{k}} ; k=1, \ldots, r\right\}$.

Now assume that $\lambda g$ is projectively induced for all $\lambda>0$. Then we have $c_{0}=0$, so that $\operatorname{dim} \mathfrak{s}_{\left(\alpha_{l}-\alpha_{k}\right) / 2}=0$ for all $1 \leq k<l \leq r$. In this case, we see that $\mathfrak{s}$ is a direct sum of ideals $\mathfrak{s}_{k}:=j \mathfrak{s}_{\alpha_{k}} \oplus \mathfrak{s}_{\alpha_{k} / 2} \oplus \mathfrak{s}_{\alpha_{k}}(\bar{k}=1, \ldots, r)$, which correspond to the hyperbolic spaces $\mathbb{C} H^{n_{k}}$ with $n_{k}=1+\left(\operatorname{dim}_{\alpha_{k} / 2}\right) / 2([\mathrm{PS} 69$, pp. 52-53]). Therefore the Lie algebra $\mathfrak{s}$ corresponds to the direct product $\mathbb{C} H^{n_{1}} \times \cdots \times \mathbb{C} H^{n_{r}}$, which is biholomorphic to $\Omega$ because the homogeneous domain $\Omega$ also corresponds to $\mathfrak{s}$. Hence (4) holds and Theorem 4 is verified.

\section{REFERENCES}

[AD03] D. A. Alekseevsky and A. J. Di Scala, Minimal homogeneous submanifolds of symmetric spaces, Lie Groups and Symmetric Spaces, AMS Translations-Series 2, 210 (2003), pp. 11-25.

[AD04] D. A. Alekseevsky and A. J. Di Scala, The normal holonomy group of Kähler submanifolds, Proc. London Math. Soc. (3), 89 (2004), pp. 193-216.

[AR95] J. ARAZY, A Survey of Invariant Hilbert Spaces of Analytic Functions on Bounded Symmetric Domains, Contemporary Mathematics Volume 185, 1995.

[Ar50] N. Aronszajn, Theory of reproducing kernels, Trans. Amer. Math. Soc., 68 (1950), pp. 337-404.

[Bo47] S. Bochner, Curvature in Hermitian metric, Bull. Amer. Math. Soc., 53 (1947), pp. 179195.

[Ca53] E. CAlabi, Isometric Imbeddings of Complex Manifolds, Ann. Math., 58 (1953), pp. 123.

[CGR90] M. Cahen, S. Gutt, And J. H. Rawnsley, Quantization of Kähler manifolds I: Geometric interpretation of Berezin's quantization, JGP, 7 (1990), pp. 45-62. 
[CGR93] M. CAhen, S. Gutt, And J. H. Rawnsley, Quantization of Kähler manifolds II, Trans. Amer. Math. Soc., 337 (1993), pp. 73-98.

[D90] M. DAJCZER, Submanifolds and isometric immersions, Mathematics Lecture Series, 13. Publish or Perish, Inc., Houston, TX, 1990.

[DDZ85] J. E. D'Atri, J. Dorfmeister, J. Zhao, And Yan Da, The isotropy representation for homogeneous Siegel domains, Pacific J. Math., 120:2 (1985), pp. 295-326.

[DS02] A. J. Di Scala, Minimal homogeneous submanifolds in Euclidean spaces, Ann. Glob. Anal. Geom., 21 (2002), pp. 15-18.

[DL07] A. J. Di SCAla And A. LOI, Kähler maps of Hermitian symmetric spaces into complex space forms, Geom. Dedicata, 125 (2007), pp. 103-113.

[DC92] M. P. Do CARmo, Riemannian geometry, Mathematics: Theory \& Applications, Birkhäuser Boston, Inc., Boston, MA, 1992.

[D85] J. Dorfmeister, Simply transitive groups and Kähler structures on homogeneous Siegel domains, Trans. Amer. Math. Soc., 288 (1985), pp. 293-305.

[DN88] J. Dorfmeister And K. NAKajima, The fundamental conjecture for homogeneous Kähler manifolds, Acta Math., 161:1-2 (1988), pp. 23-70.

[FT99] J. Faraut and E. G. F. Thomas, Invariant Hilbert spaces of holomorphic functions, Journal of Lie Theory, 9 (1999), pp. 383-402.

[199] H. IsHI, Representations of the affine transformation groups acting simply transitively on Siegel domains, J. Funct. Anal., 167:2 (1999), pp. 425-462.

[I11] H. IsHI, Unitary holomorphic multiplier representations over a homogeneous bounded domain, Adv. Pure Appl. Math., 2 (2011), pp. 405-419.

[LOI06A] A. LoI, Calabi's diastasis function for Hermitian symmetric spaces, Diff. Geom. Appl., 24 (2006), pp. 311-319.

[LZ11] A. LoI AND M. ZEDDA, Kähler-Einstein submanifolds of the infinite dimensional projective space, Math. Ann., 350 (2011), pp. 145-154.

[Na86] K. Nakajima, On j-algebras and homogeneous Kähler manifolds, Hokkaido Math. J., 15:1 (1986), pp. 1-20.

[PS69] I. I. PiatetSkit-Shapiro, Automorphic functions and the geometry of classical domains, Gordon and Breach, New York, 1969.

[TA78] M. TAKEUCHI, Homogeneous Kähler submanifolds in projective spaces, Japan J. Math, 4 (1978), pp. 171-219. 
A. J. DI SCALA, H. ISHI AND A. LOI 